\title{
THE OSMOTIC MECHANISM OF THE CUTTLEBONE
}

\author{
By E. J. Denton, J. B. Gilpin-Brown` AND J. V. Howarth \\ The Plymouth Laboratory
}

(Text-figs. I-6)

The cuttlefish can vary its density by moving liquid into or out of its cuttlebone and so varying the volume of the gas space which the cuttlebone contains. The gas pressure is always less than atmospheric and falls when liquid is pumped from the bone and there can be no question of this gas pressure balancing the external hydrostatic pressure of the sea (Denton \& GilpinBrown, I $196 \mathrm{I} a$ ). Since the cuttlebone is not an impermeable structure some other mechanism must be used for the pumping of liquid. This communication shows that osmotic forces are probably used to control the volume of the gas space within the cuttlebone.

The great defect of the swimbladder of a fish as a buoyancy mechanism is that, since the swimbladder wall is not rigid, its volume changes greatly with depth (Moreau, I876). The fish is in a state of unstable equilibrium for if it rises in the sea the swimbladder will expand and tend to push it still higher whilst if it goes deeper in the sea the swimbladder will be compressed and the fish will tend to sink more and more quickly. Here we show that within the cuttlebone the volume of the gas space remains approximately constant with change in external pressure and that it will therefore act as a buoyancy mechanism which is almost independent of depth.

\section{Material MATERIAL AND METHODS}

Experiments on freshly caught Sepia officinalis (L.) were made aboard R.V. 'Sarsia' in the spring of 1959 and the spring of 1960 . At this time of year the animals are very rarely found in the English Channel off Plymouth in depths of less than 35 fathoms and we can therefore be fairly sure that the animals used, which were taken at about 40 fathoms, had not recently migrated there from shallower water. As soon as possible after the trawl came inboard the animals were placed in sea-water circulation in the ship's tanks. Animals not used at sea were kept in two large cages in the large sea-water reservoir of the Plymouth Laboratory.

\footnotetext{
* Present address: Department of Zoology, The University, Auckland, New Zealand.
} 


\section{Extraction of liquids}

Blood was taken from the cephalic aorta.

To extract liquid from the cuttlebone the following method was used. A cuttlebone was dissected out quickly and the yellowish membrane covering the siphuncular surface was gently removed. The bone was then placed, ventral side upwards, in a bath of liquid paraffin inside a vacuum desiccator. The pressure within the desiccator was gradually reduced and the liquid which came through the siphuncular surface trickled down to collect in the cup formed by the curved posterior dorsal part of the bone (see Fig. I). When sufficient liquid had collected, atmospheric pressure was restored and the liquid removed by Pasteur pipette. Successive samples of liquid from inside the cuttlebone were obtained by progressively reducing the external pressure on the bone.

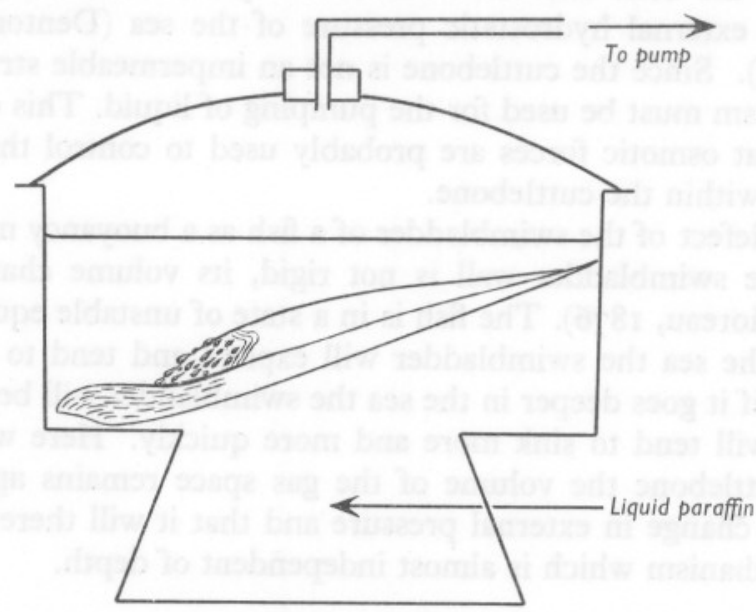

Fig. I. Apparatus used to extract liquid from within the cuttlebone. Drops of liquid can be seen coming from the siphuncular surface of the bone.

The samples of blood, sea water, and cuttlebone liquid which were to be used for later analysis were stored under liquid paraffin, in sealed tubes which were placed in sealed Kilner jars in a deep freeze. Kilner jars were used so that the samples could be later brought back to room temperature without condensation of water on the tubes containing samples.

Determinations of osmotic pressure and chemical analyses

Osmotic pressures were determined by two methods. In 1959 the freezingpoint depression was determined by a method similar to that used by Freeman \& Rigler (I957). In I960 the thermoelectric method of Hill and Baldes was used: the chamber used was similar to that described by Krogh (1939) but, 
at Dr Baldes's suggestion, this was placed not in a water bath but in air in a vacuum flask. Both methods gave results which were reproducible to $2 \%$ of the tonicity of sea water.

Analyses of chloride were made using the micro-diffusion method of Conway (1940); sodium and potassium were measured with an EEL flame photometer. In all analyses the unknown solutions were compared with sea water. The sea water used as a standard was usually that taken at sea close to the place where the animals were caught. Since, the sea water in the reservoirs of the Plymouth Laboratory usually differs a little from that at sea, this was used as a standard when studying animals which had been kept for some time in the reservoirs.

The effect of pressure on the buoyancy of the cuttlefish

A small cuttlefish was placed in a very strong metal cylinder of volume I250 $\mathrm{ml}$. designed to contain an instrument used for work in the deep sea. The cylinder was closed at one end by a thick Perspex lid and connected by a tube going through this lid to a pump and a manometer. The cylinder and

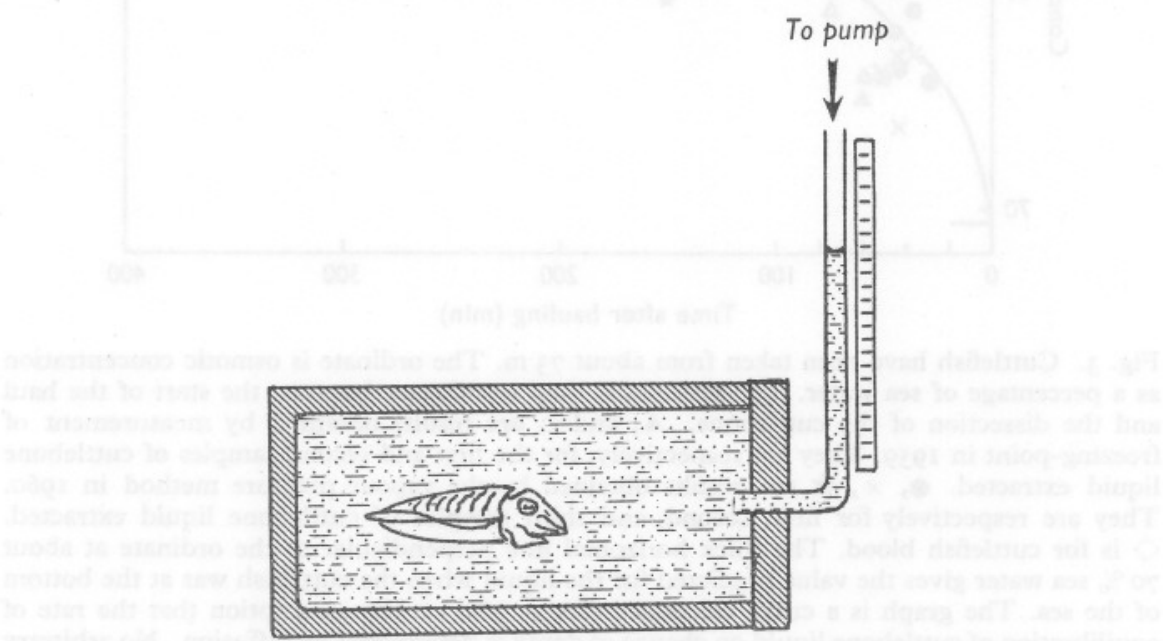

Fig. 2. Apparatus used to study the effect of pressure on the volume of the gas spaces within a cuttlefish and a pollack.

part of the tube were filled with sea water (Fig. 2) and changes in the volume of the contents of the cylinder with pressure could be measured in terms of the height of the sea water in the tube. Similar measurements were made on a young pollack. 
Osmotic pressure

RESULTS

Both in 1959 and 1960 the animals were trawled in about $73 \mathrm{~m}$. The results obtained using the depression of freezing-point in 1959 and the vapour pressure method of Hill and Baldes agreed very well; they are shown

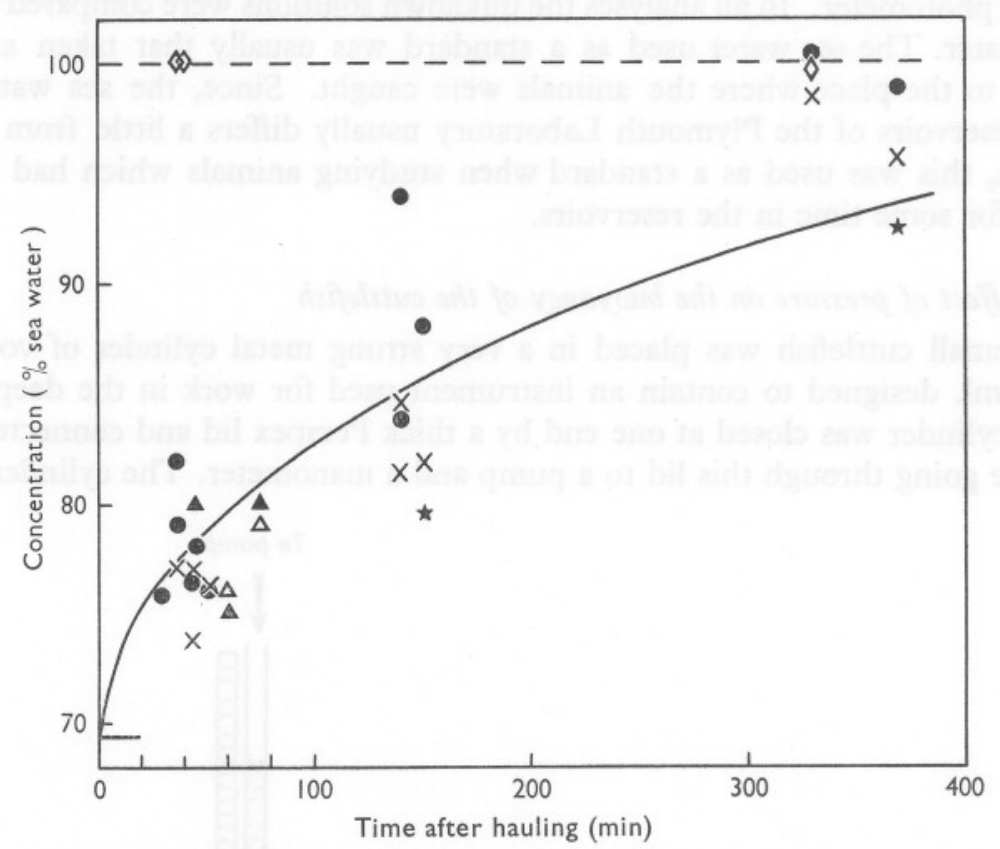

Fig. 3. Cuttlefish have been taken from about $73 \mathrm{~m}$. The ordinate is osmotic concentration as a percentage of sea water. The abscissa is time in minutes between the start of the haul and the dissection of the cuttlebone. $\boldsymbol{\Delta}$, and $\Delta$ are results obtained by measurement of freezing-point in 1959. They are respectively, for the first and second samples of cuttlebone liquid extracted. $\bullet, \times, \star$ are results obtained by the vapour pressure method in 1960 . They are respectively for first, second, and third samples of cuttlebone liquid extracted. $\diamond$ is for cuttlefish blood. The thick horizontal line perpendicular to the ordinate at about $70 \%$ sea water gives the value predicted for the liquid when the cuttlefish was at the bottom of the sea. The graph is a curve calculated (see p. 360) on the assumption that the rate of equilibration of cuttlebone liquid on change of depth is determined by diffusion. No arbitrary constants are needed in this calculation.

on Figs. 3 and 4. It can be seen that in the first $6 \mathrm{~h}$ after capture the osmotic concentration $^{1}$ of the cuttlebone liquid changes from about $75 \%$ sea water to close to $100 \%$ sea water. When successive samples were taken from a cuttlebone the later ones i.e. those from deeper inside the bone, had the lower osmotic concentrations (Fig. 3).

1 The concentration of a liquid extracted from a cuttlebone is here given in terms of the dilution of sea water which is osmotically equivalent to it; i.e. a dilution which at the ambient temperature has the same vapour pressure, or which has the same freezing-point. This concentration will be referred to as the osmotic concentration. 
The blood always had an osmotic concentration very close to that of sea water. After keeping two cuttlefish for several weeks in shallow water in the laboratory both blood and cuttlebone liquid were within $2 \%$ of the osmotic concentration of sea water.

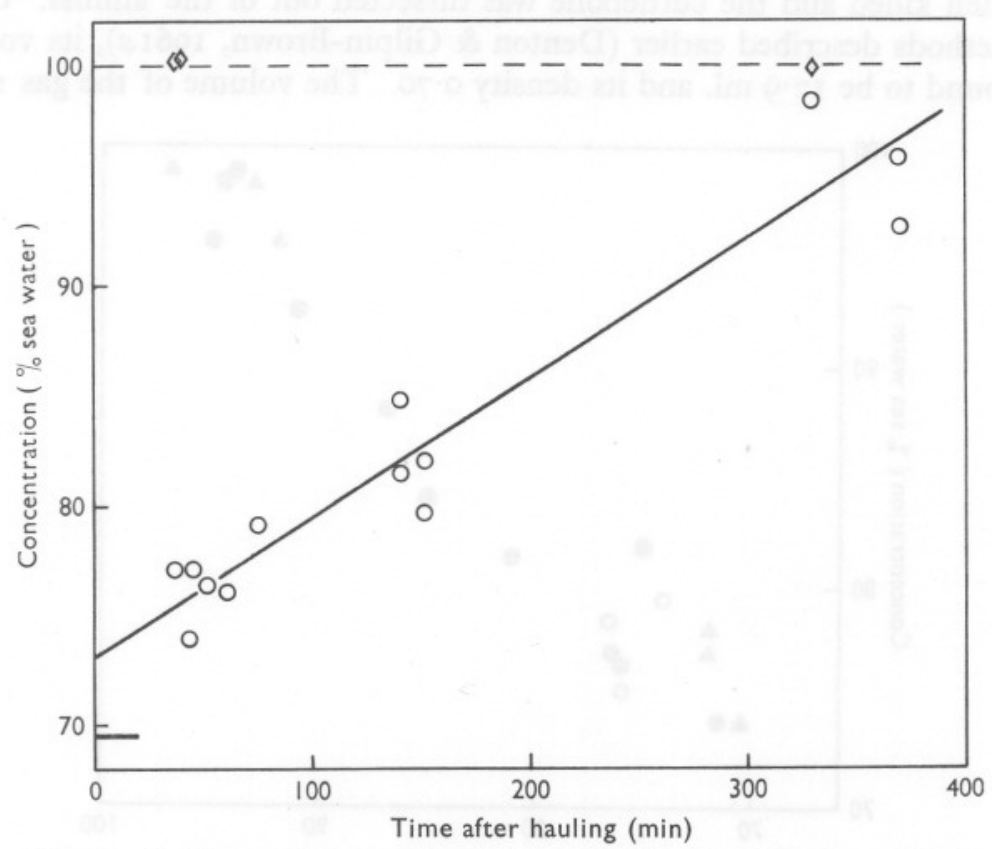

Fig. 4. The data in Fig. 3 relating to the 2 nd and 3 rd (i.e. the deeper) samples have been replotted. The minimum predicted osmotic concentration before capture is shown by a thick black line just below the $70 \%$ value on the ordinate. A straight-line extrapolation has been made back to the time when hauling began, although this is probably unfavourable to the osmotic hypothesis. It can be seen that the osmotic concentration of the cuttlebone liquid before capture must have been close to that predicted.

\section{Chemical analyses}

Fig. 5 shows that the differences in osmotic concentration between cuttlebone liquid and sea water are mainly due to reductions in the two principal ions, sodium and chloride. The concentration of sulphate which is very low in cuttlefish blood (Robertson, 1949, 1953) is also very low in cuttlebone liquid. This was shown by a qualitative test in which the precipitates given by adding acidified barium chloride solution to cuttlebone liquid and sea water were compared.

Effect of pressure

Before the experiment the cuttlefish which weighed $203 \mathrm{~g}$ in air was weighed in a box filled with sea water under sea water (Denton \& Gilpin- 
Brown, $196 \mathrm{I} b)$. The weight in sea water of the cuttlefish was found to be $+0.50 \mathrm{~g}$. After subjecting the animal to the cycle of positive pressures described below and then returning to atmospheric pressure its weight in sea water was found to be $+0.57 \mathrm{~g}$ and thus little changed. The cuttlefish was then killed and the cuttlebone was dissected out of the animal. Using the methods described earlier (Denton \& Gilpin-Brown, I96I $a$ ), its volume was found to be $17.9 \mathrm{ml}$. and its density 0.70 . The volume of the gas space

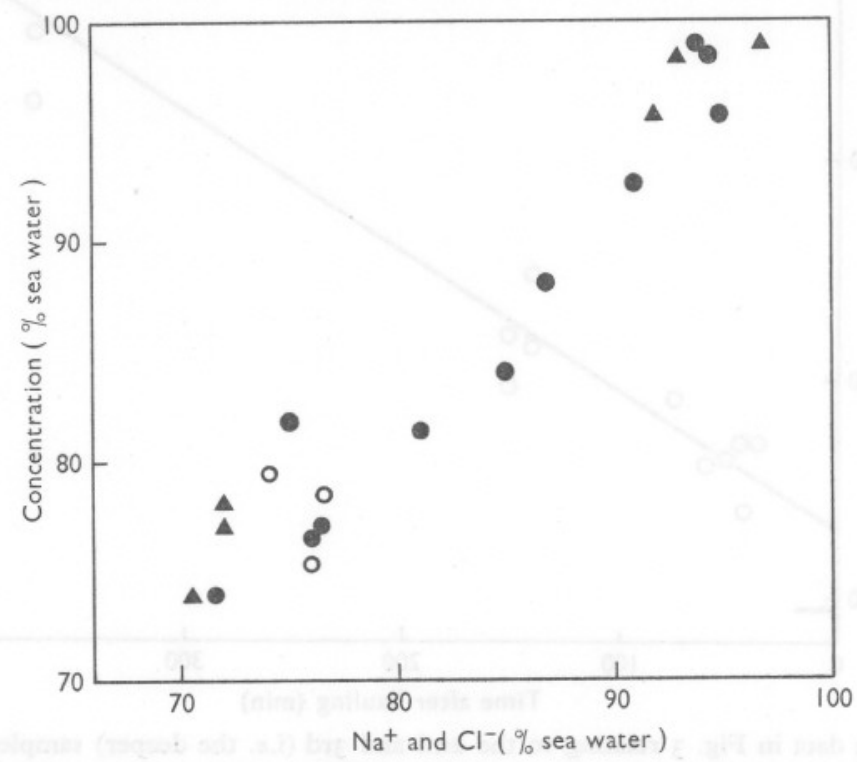

Fig. 5. Analyses made on cuttlebone liquids of various osmotic concentrations. The abscissa shows sodium and chloride concentrations as a percentage of sea water. O, Sodium (I959 samples); $\boldsymbol{\theta}$, sodium (I960 samples); $\mathbf{\Delta}$, chloride (I960 samples). The ordinate is the osmotic concentration as a percentage dilution of sea water.

was estimated as being $9.3 \mathrm{ml}$. In the living animal pressure was applied in steps of $\mathrm{I}$ atm to a pressure of $5 \mathrm{~atm}$ above atmospheric. The animal was left at this pressure for $15 \mathrm{~min}$ and then decompressed quickly. The animal was in the pressure chamber for about $40 \mathrm{~min}$. The changes in volume with pressure were almost the same for the cylinder containing the cuttlefish and the cylinder containing only sea water. The maximum change attributable to the cuttlefish was less than $0.13 \%$ of the estimated volume of the gas space in the cuttlebone.

For comparison the volume changes of a young pollack weighing $24.5 \mathrm{~g}$ were measured for the same cycle of pressures in the same apparatus. Very appreciable changes in volume were now found. We may safely assume that the very compressible element in a pollack is the gas-filled swimbladder which does not have a rigid wall like that of the cuttlebone. On plotting volume 
change against I/pressure a straight line is given and, by extrapolating back to $\mathrm{I} / \mathrm{p}=0$ where the volume would be zero, we can estimate the volumes of this gas at other pressures. The volume of gas at one atmospheric pressure corresponded to $4.6 \%$ of the volume of the fish. This is close to the value expected for this fish had lived for some time in shallow water in the aquarium before the experiment.

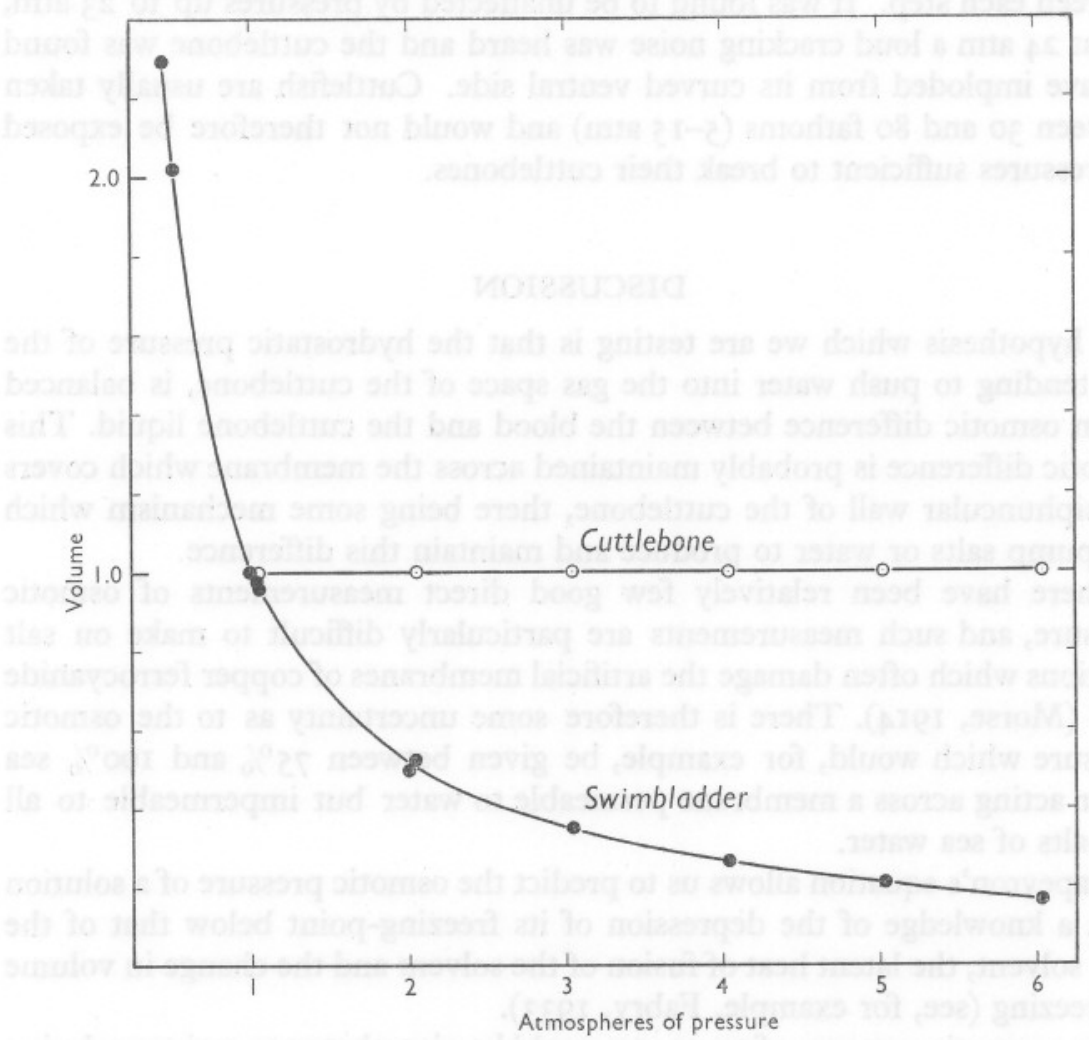

Fig. 6. The change in volume of gas space with pressure: $\bigcirc$, in the cuttlebone; in the swimbladder of a pollack. The volumes of the gas spaces at one atmosphere pressure and room temperature are taken as unity.

Fig. 6 shows both the change in volume of the cuttlebone gas-space (in the living cuttlefish) when the pressure is changed and also the volume change of the gas in the swimbladder of the pollack. The volumes are given with respect to the volumes at I atm pressure and room temperature. Since the volume of the gas space of the cuttlebone was about eight times that of the gas in the fish's swimbladder there can be no question that the method was sensitive enough to detect any but the smallest changes in the volume of the gas space in the cuttlebone. 


\section{Effects of high pressures on the cuttlebone}

Mr R. Dobson of the National Institute of Oceanography kindly tested the effect of pressure on a cuttlebone. This was placed in a closely applied polythene bag, to prevent it merely filling with water, and the pressure increased in steps of $\mathrm{I}$ atm. The cuttlebone was decompressed and examined between each step. It was found to be unaffected by pressures up to $23 \mathrm{~atm}$, but at $24 \mathrm{~atm}$ a loud cracking noise was heard and the cuttlebone was found to have imploded from its curved ventral side. Cuttlefish are usually taken between 30 and 80 fathoms $(5-15 \mathrm{~atm})$ and would not therefore be exposed to pressures sufficient to break their cuttlebones.

\section{DISCUSSION}

The hypothesis which we are testing is that the hydrostatic pressure of the sea, tending to push water into the gas space of the cuttlebone, is balanced by an osmotic difference between the blood and the cuttlebone liquid. This osmotic difference is probably maintained across the membrane which covers the siphuncular wall of the cuttlebone, there being some mechanism which can pump salts or water to produce and maintain this difference.

There have been relatively few good direct measurements of osmotic pressure, and such measurements are particularly difficult to make on salt solutions which often damage the artificial membranes of copper ferrocyanide used (Morse, I9I4). There is therefore some uncertainty as to the osmotic pressure which would, for example, be given between $75 \%$ and $100 \%$ sea water acting across a membrane permeable to water but impermeable to all the salts of sea water.

Clapeyron's equation allows us to predict the osmotic pressure of a solution from a knowledge of the depression of its freezing-point below that of the pure solvent, the latent heat of fusion of the solvent and the change in volume on freezing (see, for example, Fabry, 1933).

If an osmotic pressure of $22.4 \mathrm{I}$ atm could be given between a given solution and water at $0^{\circ} \mathrm{C}$, then Clapeyron's equation would predict that the freezingpoint of this solution would be $-\mathrm{I} \cdot 85^{\circ} \mathrm{C}$ (Morse, I9I4) [22.4I atm is the pressure exerted by a gram molecule of a perfect gas with a volume of $\mathrm{I} 1$. at $0^{\circ} \mathrm{C}$ ]. From a knowledge of the osmotic pressure at the freezing-point we may predict the osmotic pressure at another temperature by assuming that it is proportional to the absolute temperature. This assumption is reasonably sound for temperatures close to that of the freezing-point. Morse showed that a solution of cane sugar with a freezing-point depression of $\mathrm{I} .829^{\circ} \mathrm{C}$ gave at $0^{\circ} \mathrm{C}$ an osmotic pressure (against water) of $22.13 \mathrm{~atm}$ (calculated value $2 \mathrm{I} .99 \mathrm{~atm}$ ), whilst at $15^{\circ} \mathrm{C}$ the osmotic pressure was $23.3 \mathrm{I} \mathrm{atm}$ (calculated value $23.20 \mathrm{~atm}$ ); above $25^{\circ} \mathrm{C}$ he found that the osmotic pressures 
were greater than those predicted. We shall assume: (I) that since the freezing-point depression of the standard sea water used was $-\mathrm{I} \cdot 9 \mathrm{I}^{\circ} \mathrm{C}$ instead of $-\mathrm{I} \cdot 85^{\circ} \mathrm{C}$ (its salinity was $35.25 \%$ ) the osmotic pressure between it and pure water would be $(22 \cdot 4 \mathrm{I} \times \mathrm{I} \cdot 9 \mathrm{I}) / \mathrm{I} \cdot 85$, i.e. $23 \cdot \mathrm{I}$ atm at $0^{\circ} \mathrm{C} ;(2)$ where we have solutions of two different freezing-points the osmotic pressure which would exist between them is proportional to the difference in freezing-point depressions, for example between solutions of freezing-point depressions $\mathrm{I} \cdot 9 \mathrm{I}^{\circ} \mathrm{C}$ and $\mathrm{I} \cdot 39^{\circ} \mathrm{C}$ the osmotic pressure difference at $0^{\circ} \mathrm{C}$ would be $[23 . \mathrm{I} \times(\mathrm{I} \cdot 9 \mathrm{I}-\mathrm{I} \cdot 39)] / \mathrm{I} \cdot 9 \mathrm{I} \mathrm{atm}$; and (3) the osmotic pressure difference between two solutions is proportional to the absolute temperature. At $10^{\circ} \mathrm{C}$, the temperature of the sea where the animals were caught, the osmotic pressure difference between sea water and pure water would be $24.0 \mathrm{~atm}$.

In the measurements of vapour pressures we are matching our unknown solutions against diluted sea waters. Now the galvanometer deflection given between pure water and $25 \%$ sea water was very nearly the same as that between 75 and $100 \%$ sea water; this shows that the vapour pressure differences are approximately the same. We shall assume that the osmotic pressure is proportional only to difference in concentration. This means, for example, that at $10^{\circ} \mathrm{C}$ the difference in osmotic pressure between 75 and $100 \%$ sea water would be $[24.0 \times(100-75)] /$ I00 atm. The sea waters were diluted by adding known volumes of sea water to known volumes of distilled water; for example, $50 \%$ sea water was obtained by mixing equal volumes of sea water and distilled water. This method of dilution differs from that recommended by Morse who used the weight-normal system. The errors given by neglecting these differences are very small and are unfavourable to the osmotic hypothesis advanced here.

The animals used were caught in $73 \mathrm{~m}$ and for them the maximum pressure pushing water into the cuttlebone would have been 8.2 atm. Only about $0.8 \mathrm{~atm}$ of this pressure would be balanced by the gas pressure inside the cuttlebone leaving, on the present hypothesis, $7 \cdot 4$ atm to be balanced by the osmotic difference between blood and cuttlebone liquid. Since the blood has the same osmotic concentration as sea water this requires that the cuttlebone liquid should have a concentration of $69 \cdot 2 \%$ sea water. Figs. 3 and 4 show that the composition of liquid within the cuttlebone changes very appreciably in the first few hours following the hauling of the trawl. About $40 \mathrm{~min}$. after the start of the haul the samples from deeper inside the cuttlebone had osmotic concentrations of about $75 \%$ sea water, whilst $6 \mathrm{~h}$ later they were equivalent to about $97 \%$ sea water. To estimate the concentration which the liquid would have had at the bottom of the sea we may extrapolate back to the time at which the haul began. It can be seen from Fig. 4 that before capture the concentration of salts in the cuttlebone liquid would have been reasonably close to the value of $69 \cdot 2 \%$ sea water predicted. If the 
cuttlefish had been above the bottom of the sea before capture then the expected value would have been higher than $69 \cdot 2 \%$.

How can we interpret the fact that, in the first hours following capture, the liquids deeper within the cuttlebone differ from those nearer the siphuncular surface? This presumably comes about because diffusion limits the rate at which complete equilibrium can be re-established after a change in depth. Taking conditions similar to those of our experiments, let us suppose that an animal is living at $73 \mathrm{~m}$ depth and that the cuttlebone liquid is uniform in composition and of a concentration equivalent to $69 \cdot 2 \%$ sea water. The animal is then brought into very shallow water and after some time re-establishes equilibrium with a concentration of salts in the cuttlebone liquid everywhere equal to $100 \%$ sea water. Let us try to calculate the way in which this change takes place. We shall assume, for the sake of simplicity of calculation, that the volume of liquid within the cuttlebone remains constant and that the membrane covering the siphuncular surface immediately re-establishes and then maintains equilibrium between osmotic and hydrostatic pressures, i.e. it keeps the concentration of salts just inside the siphuncular surface equal to that of sea water. Until equilibrium is completely re-established the concentration of salts just inside the siphuncular surface will be higher than that deeper within the cuttlebone and salts will diffuse inwards. The membrane will continuously have to pump more salts to balance those diffusing away and thus ensure that there is no concentration difference across the membrane itself. Now the liquid within the cuttlebone is confined in very narrow channels by the lamellae, and mixing will not readily take place. The exchange of salts between the liquid just inside the siphuncular surface and that deeper within the cuttlebone will be limited by diffusion. This surface has an area of about $16 \mathrm{~cm}^{2}$ in an average-sized cuttlebone and on the average about $3 \mathrm{ml}$. of liquid were extracted from a bone. Allowing for the great inclination of the siphuncular surface to the lamellae and for the solid matter of the cuttlebone we arrive at a depth of liquid along the channels of about $0.75 \mathrm{~cm}$. We are only concerned here with the liquid extracted under reduced pressure from the siphuncular surface.

This problem of the change in distribution of salts within the lamellae is roughly similar to a problem in diffusion solved by Hill (I928, pp. 55-69). $\mathrm{He}$ considers the diffusion of oxygen into a parallel-sided plane sheet of thickness $b$, bounded on one side by an impermeable wall and exposed on the other wall at time $t=0$ to a maintained change in concentration of oxygen. He gives (his Table 3 ) the average saturation of the sheet with oxygen for various times following this change. By substituting, in his equations, the value for the diffusion constant of the salts in sea water, here taken as equal to the $\mathrm{I} \cdot 39 \times 1 \mathrm{I}^{-5} \mathrm{~cm}^{2} / \mathrm{sec}$ given for sodium chloride in water (International Critical Tables, cited by Höber, 1945) and $0.75 \mathrm{~cm}$ for $b$, we can find the way in which the average concentration of salts within the 
cuttlebone will change with time following a change in depth. This is shown by the curve of Fig. 3 .

It can be seen that the times which the calculation predicts for given changes in concentration of salts within the cuttlebone are in fair agreement with those found by experiment. After about $60 \mathrm{~min}$ the average concentration of salts would have risen from $69 \cdot 2$ to $80 \%$ of sea water, at $250 \mathrm{~min}$ to about $90 \%$ of sea water.

The explanation given above is certainly too simple, for it seems certain that some net exchanges of water take place in the first few hours following change in depth. We expected that, on coming to the surface and so reducing the hydrostatic pressure, the osmotically stronger blood would draw water from the cuttlebone liquid. In the experiments we were surprised to find that greater volumes of liquid could be extracted from the cuttlebones of cuttlefish taken $2 \mathrm{~h}$ after capture than those used in the first hour after capture.

Nevertheless, whatever the actual exchanges of salt and water which take place between cuttlebone and blood on change in depth of the cuttlefish, it seems certain enough that the slowness of diffusion of salts within the cuttlebone liquid will greatly limit the rate of equilibration. This is clearly a very advantageous arrangement for the cuttlefish. If, for example, it swims upwards and stays for I or $2 \mathrm{~h}$ near the surface of the sea, a small exchange of salt or water across the siphuncular membrane can re-establish the balance across the siphuncular wall between osmotic and hydrostatic pressures. The buoyancy of the cuttlefish need hardly change. The liquid deeper inside the cuttlebone will preserve a concentration close to that suitable for the bottom of the sea, where the cuttlefish probably spends most of its life. When the animal returns to the bottom of the sea very little work will have to be done to re-establish complete equilibrium.

We may give a numerical example; consider a cuttlefish at $70 \mathrm{~m}$ (i.e at 8 atm pressure) in which all the liquid inside the cuttlebone is osmotically equivalent to about $70 \%$ sea water. The animal now swims to the surface (I atm pressure). The liquid just inside the siphuncular wall is made osmotically equivalent to $100 \%$ sea water and a balance between osmotic and hydrostatic pressures across the siphuncular wall is restored. Owing to the slowness of diffusion, liquid far from the siphuncular wall retains for several hours a concentration approximately equivalent to $70 \%$ sea water and will not have to be changed when this animal swims down again to the sea bed.

This buoyancy system would be expected to be relatively insensitive to changes in depth and to compare very favourably with that of the swimbladder of a fish where changes in depth, particularly those near the surface, produce great changes in the volume of the gas space of the swimbladder and hence of the fish's buoyancy. This is what the experiment on pressure showed to be true.

An osmotic difference between cuttlebone liquid and cuttlefish blood can hold water out of the cuttlebone, but it cannot balance the crushing effect of 
the pressure of the sea. The cuttlebone is, however, very strong. Its structure, with plates held apart by numerous pillars, is especially well suited to sustain compression and we find that it only breaks under pressures greater than those found in the animal's normal depth range.

We wish to thank Dr T. I. Shaw for very helpful discussions and Mr F. G. C. Ryder for making the apparatus used for vapour pressure determinations.

\section{SUMMARY}

Experiments are described which test the hypothesis that the cuttlefish controls the relative volumes of gas space and liquid within its cuttlebone by an osmotic mechanism acting across the siphuncular surface of the bone. When the animal is at the bottom of the sea it would maintain the gas space within the cuttlebone by balancing the hydrostatic pressure of the sea by an osmotic force between the liquid within the cuttlebone and the blood.

In cuttlefish kept for some weeks in shallow water in an aquarium all the liquid taken from the cuttlebone is almost isotonic with the animals' blood.

In animals recently hauled from the bottom of the sea the cuttlebone liquid is markedly hypotonic to sea water and hence to the blood.

These lower osmotic concentrations are given chiefly by reduction in the concentrations of the sodium and chloride ions.

After bringing animals up from about $70 \mathrm{~m}$ to the surface of the sea the osmotic concentration of the cuttlebone liquid rises from about $75 \%$ of sea water some $40 \mathrm{~min}$ after starting to haul the trawl, to about $97 \%$ of sea water $6 \mathrm{~h}$ later. Extrapolation back to the time at which hauling began gives a concentration of salts close to that predicted by the osmotic hypothesis.

Whilst the cuttlebone liquid is increasing in ionic concentration the liquid deeper in the cuttlebone is hypotonic to that just inside the siphuncular surface. This is explained in terms of the slowness of exchange of salts along the narrow channels between the lamellae of the cuttlebone. On the assumption that diffusion limits this exchange the magnitude of the effect is calculated and shown to be in fair accord with the experimental results.

In the living cuttlefish external pressure, at least up to $6 \mathrm{~atm}$, has little or no effect on the volume of the gas space of the cuttlebone and the density of the cuttlefish will not change appreciably with change in depth.

The cuttlebone is very strong and can withstand high external pressures. 


\section{REFERENCES}

Conway, E. J., 1940. Micro-diffusion Analysis and Volumetric Error. 306 pp. New York: van Nostrand.

Denton, E. J. \& Gilpin-Brown, J. B., I96I $a$. The buoyancy of the cuttlefish, Sepia officinalis (L.). F. mar. biol. Ass. U.K., Vol. 4I, pp. 319-342.

- I $96 \mathrm{I} b$. The distribution of gas and liquid within the cuttlebone. F. mar. biol. Ass. U.K., Vol. 4I, pp. 365-38r.

FABRY, C., I933. Éléments de Thermodynamique. 216 pp. Paris: Armand Colin.

Freeman, R. F. H. \& RigleR, F. H., I957. The responses of Scrobicularia plana (Da Costa) to osmotic pressure changes. F. mar. biol. Ass. U.K., Vol. 36, pp. 55367.

HiLl, A. V., 1928. The diffusion of oxygen and lactic acid through tissue. Proc. roy. Soc. B, Vol. 104, pp. 39-96.

HöвER, R., 1945. Physical Chemistry of Cells and Tissues. 676 pp. London: J. and A. Churchill.

Krogh, A., 1939. Osmotic Regulation in Aquatic Animals. 242 pp. Cambridge Comparative Physiological Series, Cambridge University Press.

MOREAU, A., I876. Recherches éxpérimentales sur les fonctions de la vessie natatoire. Ann. Sci. nat. (Zool.), Sér. 6, T. 4, No. 9, 85 pp.

Morse, H. N., 1914. The Osmotic Pressure of Aqueous Solutions. 222 pp. Washington: Carnegie Institution.

ROBERTSON, J. D., 1949. Ionic regulation in some marine invertebrates. F. exp. Biol., Vol. 26, pp. 182-200.

1953. Further studies on ionic regulation in marine invertebrates. F. exp. Biol., Vol. 30, pp. 277-96. 